\title{
Eriksen flanker delta plot shapes depend on the stimulus
}

\author{
Michael S. Pratte ${ }^{1}$ \\ Accepted: 18 September 2020 / Published online: 5 November 2020 \\ (C) The Psychonomic Society, Inc. 2020
}

\begin{abstract}
Several experimental paradigms are purported to measure response conflict, including the Stroop, Simon, and Eriksen flanker tasks. Although these tasks are often treated as being similar, delta plot analyses of response time distributions have revealed marked differences across them. Several theories have been proposed to explain these differences, however, assessing their veracity is difficult given the numerous differences across tasks. To explore what might cause delta plots to differ in a more controlled manner, here stimulus materials were manipulated across four Eriksen flanker tasks. The results reveal substantially different delta plot shapes for different stimuli: positive-going functions when color or motion served as the target and flankers, and delta plots with negative-going components when stimuli were arrows or orientated gratings. These results cast doubt on the proposal that negative-going delta plots occur only when spatial location serves as the interfering stimulus dimension. Moreover, because targets and flankers were always of the same stimulus type, the results also suggest that differences in materials across the relevant and irrelevant dimensions do not determine delta plot shapes. Instead, we propose that the delta plot shape is determined by several factors, including how early the interfering information is processed in the visual cortex.
\end{abstract}

Keywords Conflict tasks · Inhibition · Interference $\cdot$ Stroop · Simon

Many different tasks have come to be collectively known as conflict tasks, as they all appear to measure how the cognitive system deals with conflicting information. For example, in the Stroop task (Stroop, 1935) the target information is color and the conflicting information is color word names (Fig. 1a). When the to-be-reported color matches the word name (congruent condition), responses are faster than when they mismatch (incongruent condition). In the Simon task (Simon, 1969) participants must identify the color of a stimulus that is shown on either the left or right side of the display (Fig. 1b). Responses are faster when the stimulus is shown on the same side of the display as the correct response hand, and slower when the stimulus location and response hand conflict. In both the Stroop and Simon tasks the target information is color, but in the Stroop task the conflicting information is word meaning, and in the Simon task it is spatial location. Whereas the target and interfering information in these tasks are therefore of different modalities, in other conflict tasks the target

Michael S. Pratte

prattems@gmail.com

1 Mississippi State University, P.O. Box 6161, Mississippi State, MS, 39762, USA and distracting information can be of the same type. For example, in the Eriksen flanker task (Eriksen \& Eriksen, 1974; Eriksen, 1995) the target is a letter centered on the display, and the distractors are also letters which flank the target, and can either match or mismatch the target letter identity (Fig. 1c). Such differences between these and other conflict tasks have been studied extensively (Kornblum, Hasbroucq, \& Osman, 1990), however, these tasks are often used interchangeably as generic tools to measure how the cognitive system deals with conflicting information.

Although it seems reasonable to view the Stroop, Simon and Eriksen tasks as different flavors of conflict paradigms, recent work using distributional analyses of response times has revealed fundamental differences in how conflict manifests across them. In particular, in the delta plot analysis (De Jong, Liang, \& Lauber, 1994) congruency effects at different percentiles of the response time distributions are plotted against the mean response time of each percentile. This plot characterizes how the congruency effect in response time depends on the overall latency of responses. For example, the positive-going line in Fig. 2 is the delta plot of a Stroop effect. Delta plots of Stroop effects are almost always positive-going, which has been taken to suggest that the effect of word meaning on color identification is small for fast responses and grows 


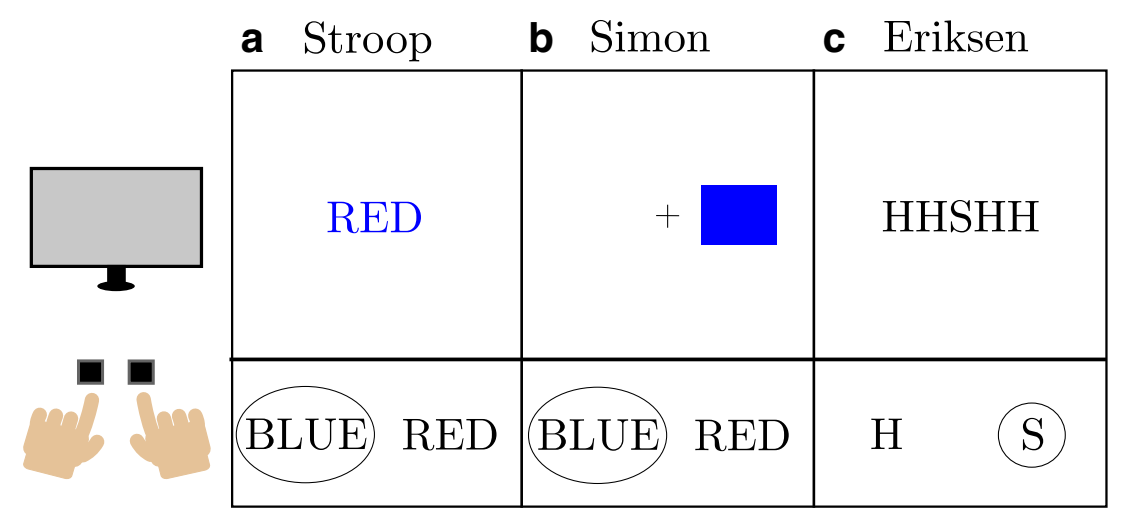

Fig. 1 Examples of Stroop (a), Simon (b), and Eriksen (c) conflict tasks. Each example stimulus display (top) shows an incongruent trial. The correct response of the two options (bottom) is circled

as overall responses slow. This pattern holds for nearly all other conflict tasks (see Pratte, Rouder, Morey, \& Feng, 2010), including some versions of the Eriksen flanker task (Ridderinkhof, Scheres, Oosterlaan, \& Sergeant, 2005; Burle, Spieser, Servant, \& Hasbroucq, 2014). The Simon effect, however, typically shows the opposite pattern: Simon effect delta plots are negative-going, such as that shown in Fig. 2. This pattern implies that the interfering effect of spatial location is largest for the fastest responses and decreases over time.

Several theories have been proposed to explain why Simon delta plots have a negative-going trajectory. In one prominent example, Ridderinkhof (2002a, b) suggests that there is a fast, automatic activation of the irrelevant spatial location, which leads to interference effects when responses are fast. Over time this interfering information is thought to be actively suppressed, causing it to have less influence

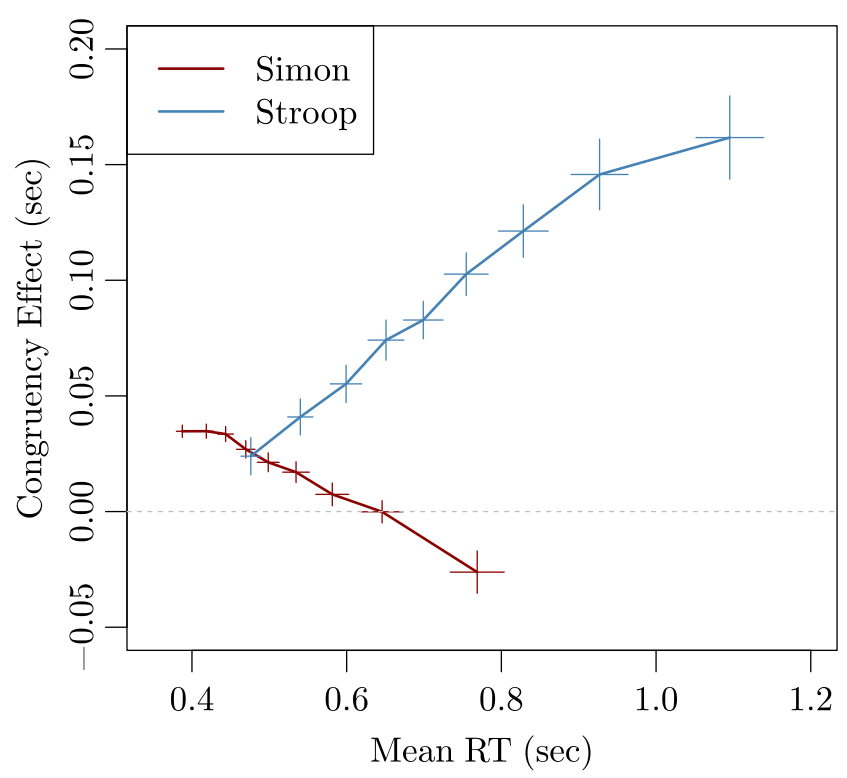

Fig. 2 Delta plots of Stroop and Simon effects from Pratte et al. (2010) when responses are slower (see also Hommel, 1993). This activation-suppression model implies that the downward trajectory of Simon delta plots reflects the suppression of interfering spatial location information, and that the degree to which a delta plot trends downward provides a measure of the magnitude of suppression. This logic has been used in numerous studies to characterize how the strength of suppression varies across conditions and populations. However, the delta plots for nearly all other conflict tasks are positive-going, and it is not clear how the activationsuppression model can account for such results: why would there be little suppression of irrelevant information in tasks that exhibit positive-going delta plots, such as the Stroop task? One possibility is that the Simon effect is unique because spatial location serves at the interfering stimulus dimension, and visual space may be processed differently than other types of interfering information (Wiegand \& Wascher, 2005; Pratte et al., 2010). Left/right location in particular may have particular processing characteristics, given the strong left-right lateralization of visual space and its neural representation across hemispheres. There has been some evidence for this explanation, such as the finding that delta plot slopes are positive rather than negative in a Simon task if stimuli and responses are located along the vertical rather than the horizontal dimension (Wiegand \& Wascher, 2005). Likewise, whereas identifying the words "left" and "right" to the left or right of fixation produces a negativegoing delta plot, the words "above" and "below" positioned above or below fixation, with response buttons in an vertical configuration, produces a positive-going delta plot (Pratte et al., 2010).

Whereas the left/right lateralization account merely suggests that something about interfering left/right spatial location is unique, Ulrich, Schröter, Leuthold, and Birngruber (2015) proposed a more general explanation for why some delta plots go up and some down. In their formal model, a version of the drift diffusion model, activation of the target and interfering information grows independently over time, 
until the sum of their activity reaches a threshold causing a response. Critically, in this model the speed with which activation of the irrelevant information grows over time and then decays (or is inhibited) is free to vary across tasks. Fitting the model to data from a Simon task suggested that the interfering spatial location information grows rapidly, and then decays rapidly, relative to accumulation of the target color information. This model accurately accounted for the typical negative-going Simon delta plot, leading Ulrich et al. (2015) to suggest that negative-going delta plots reflect situations in which the interfering information accumulates and then diminishes rapidly relative to the accumulation of target information. Alternatively, fitting the model to data from an Eriksen flanker task suggested that the interfering flanker information accumulates more slowly and decays more slowly, relative to the accumulation of target information. Consequently, the flankers will initially have little influence on target processing, but interference grows over time as the irrelevant information accumulates, accurately characterizing the positive-going flanker delta plot observed by Ulrich et al. (2015).

Hübner and Töbel (2019) found evidence for the idea that delta plot shapes are affected by the relative time course of target and distractor information accumulation. By manipulating the time between the onset of flankers and that of the target in an Eriksen task, they found positivegoing delta plots when the flankers appeared immediately before the target, but a negative-going plot if the flankers were presented $400 \mathrm{~ms}$ before the target (see also Mattler 2003). Although this result is consistent with the idea that negative-going deltas occur when the interfering information accumulates before the target information, it might reflect other aspects of processing such as the degree to which attention is captured by the flankers before having to shift to the subsequent target. Critically, however, the delta plots had a negative-going component even though spatial location was not the interfering information, suggesting that delta plot shapes can be determined by the relative time course of processing relevant and irrelevant information, regardless of the particular stimulus materials.

Although delta plots of the Eriksen flanker effect are often positive-going when targets and flankers are presented simultaneously, this result is not as homogeneous as delta plot results for Stroop and Simon effects. This variability may be because the Eriksen task is unique in that, across studies, a wide variety of stimulus materials have been used as targets and interfering stimuli. For example, in addition to the letters originally used by Eriksen and Eriksen (1974), stimuli in the flanker task have included color (Rafal et al., 1996), visual shapes such as arrows (Kopp, Rist, \& Mattler, 1996), mid-level visual features like biological motion (Thornton \& Vuong, 2004), and higher level information including emotion as conveyed by faces
(Fenske \& Eastwood, 2003). The degree to which delta plot shapes change across variations in flanker stimuli has not been directly studied, but can gleaned by comparing previous studies. For example, Burle, Spieser, Servant, and Hasbroucq (2014) used letters as targets and flankers, and like Ulrich et al. (2015) observed positive-going delta plots that were highly similar to those from the Stroop task. Servant, Montagnini, and Burle (2014) also found positivegoing delta plots in a flanker task in which color was the target and flanker information (see also Davranche, Hall, \& McMorris, 2009). Alternatively, Ridderinkhof, Scheres, Oosterlaan, and Sergeant (2005) used leftwardand rightward-pointing arrows as target and flanker stimuli, and observed delta plots that initially increased but then appeared to level off. Such inverted-U-shaped delta plots are common, such as that observed for the Simon effect by Ulrich et al. (2015), and are often taken as a hallmark of late distractor suppression.

The finding that an arrow flanker task produces a similar delta plot as the Simon task is concordant with the left/right lateralization account: Leftward and rightward arrows induce reflexive shifts in spatial attention (Stevens, West, Al-Aidroos, Weger, and Pratt, 2008), which may be sufficient to cause the same sort of lateralized interference that is observed in Simon tasks and the resulting negativegoing delta plots. It is not clear, however, how other theories might account for different delta plot shapes across flanker stimulus types. For example, the activationsuppression account (Ridderinkhof 2002a, b) would require that some flanker stimuli are suppressed while others are not, although it is not clear why this might be the case. Likewise, the accumulation account (Ulrich, Schröter, Leuthold, \& Birngruber, 2015) would imply that for some stimuli the flanker information accumulates slowly relative to the target, whereas for other stimuli flanker information accumulates fast relative to target accumulation. Flankers might accumulate faster than targets, for example, if processing is additive across the two flanker stimuli (White, Ratcliff, \& Starns, 2011). Alternatively, targets might accumulate faster as they are attended and presented at the fovea where visual sensitivity is high relative to that in the periphery (Strasburger, Rentschler, \& Jüttner, 2011). It is not obvious, however, why the relative timing of target and flanker accumulation might vary depending on the stimulus type.

Although the inverted-U-shaped delta plots observed for the arrow flanker task are intriguing, participants in the Ridderinkhof et al. (2005) study were children, so it is not clear whether this result reflects the use of arrows or the adolescent participants. Similar inverted-Ushaped delta plots were observed for an arrow flanker task in another study testing adults (Wylie, Ridderinkhof, Eckerle, and Manning, 2007), however, in some studies 
the arrow flanker produced what appear to be increasing deltas (Wylie et al., 2009; White et al., 2011; Mattler, 2003). Moreover, in one study a flanker task with letters as stimuli produced what appears to be an inverted-U-shaped delta plot (Mattler, 2003). Unfortunately, none of these studies performed statistical analyses on the overall shapes of delta plots. Moreover, they did not assess how delta plot patterns might differ across flanker tasks that use different stimulus materials. Whereas a great deal of effort has been devoted to understanding why delta plots vary in shape across tasks like Stroop and Simon, these tasks differ from one another in numerous ways, making it difficult to isolate any particular cause. However, understanding how delta plots might vary across different versions of the same task, such as flanker tasks utilizing different stimulus materials, provides a powerful way to study factors that may drive the delta plot shape using systematic manipulations of task characteristics.

Here we examine how delta plot shapes of the Eriksen flanker effect depend on the type of stimulus materials that comprise the target and flankers. In Experiments 1 and 2 we replicate previous findings that for color and arrow stimuli the delta plot is positive-going and inverted-Ushaped, respectively. Although the left/right spatial location account of the Simon effect anticipates this dissociation, there is a potential confound that can also explain it: color is a mid-level visual feature that involves several brain areas, whereas spatial location is low-level visual feature that is processed early in the visual processing stream. In Experiments 3 and 4 we therefore assess flanker effects in which orientation and motion direction serve as the stimuli, and find that the level of stimulus processing provides a better account of when delta plots will be positive- or negative-going than the left/right spatial location account. Taken together, the four experiments clearly show that the time course of Eriksen flanker effects depends on the stimulus materials, providing insights into why they also differ across tasks.

\section{Experiment 1: color}

Servant et al. (2014) found that when color served as the target and flanker information in an Eriksen task, response times on incongruent trials were both slower and more variable than congruent trials, as is typical for most conflict tasks (Wagenmakers \& Brown, 2007). This pattern of increasing mean and standard deviation implies an increasing delta plot if certain conditions are met (Speckman, Rouder, Morey, \& Pratte, 2008; Zhang \& Kornblum, 1997). However, Servant et al. did not investigate delta plots, so in Experiment 1 we examine the delta plot for a color version of the Eriksen flanker task, to determine whether it is indeed positive-going.

\section{Method}

Participants Fifty-six undergraduate students at Mississippi State University participated in a one-hour session in exchange for course credit. Data from nine participants were not analyzed due to overall low accuracy on the task $(<90 \%)$, suggesting that they did not follow instructions. Participants provided informed consent prior to participation, and all studies were approved by the institutional review board at Mississippi State University.

Stimuli \& design Stimuli were presented on 24-inch LCD monitors using the Psychophysical Toolbox (Brainard, 1997; Kleiner et al., 2007; Pelli, 1997) run on Octave (Eaton, Bateman, Hauberg, \& Wehbring, 2014) on the Linux operating system. Monitors were gamma corrected using a psychophysical technique (To, Woods, Goldstein, \& Peli, 2013). Up to four people participated in the same session simultaneously, seated within semi-private cubicles within a larger room. To avoid disruption, after the main experiment participants completed a filler task (visual search) until everyone in that session completed the flanker task.

Each trial began with the presentation of a central target rectangle and two flanking rectangles located to the left and right of the target (Fig. 3a). Each rectangle subtended $1^{\circ}$ visual angle in height and $2^{\circ}$ in width. Flanker centers were located at $\pm 3^{\circ}$ from the target center, such that the target and flanker edges were separated by $1^{\circ}$. Targets and flankers were presented simultaneously for $200 \mathrm{~ms}$. Stimuli were either red $(R=1, G=0, B=0)$ or green $(R=0, G=1$, $\mathrm{B}=0)$, presented on a grey background $(\mathrm{RGB}=0.5)$, and both flankers were always the same color. A central fixation bullseye was presented throughout the experiment (black and white rings, subtending $.4^{\circ}$ ) and participants were encouraged to maintain fixation. A response period with only the fixation point followed presentation of the stimuli and remained until a response was given. Participants were instructed to ignore the flankers, and to report the color of the central target as red with their right hand ("?" key) or green with their left ("z" key). Responses were encouraged to be made as quickly as possible, while also maintaining high accuracy. Following correct responses the fixation point turned all white for $150 \mathrm{~ms}$ as positive feedback; following an incorrect trial the fixation point turned all black for $1000 \mathrm{~ms}$ as negative feedback. A $1000 \mathrm{~ms}$ inter-trial interval then preceded the start of the next trial.

Each participant completed 20 practice trials followed by 900 experimental trials. On practice trials the target and flanker colors were chosen randomly. Experimental trials 


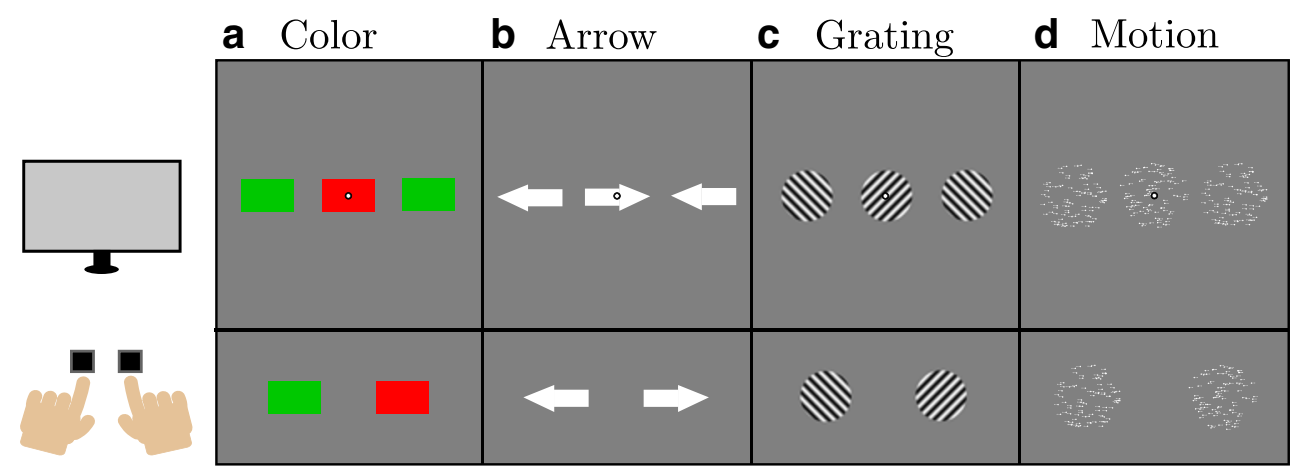

Fig. 3 Eriksen flanker tasks in Experiments 1-4. Stimuli were color in Experiment 1 (a), arrows in Experiment 2 (b), oriented gratings in Experiment 3 (c), and random-dot motion in Experiment 4 (d). The top row shows example displays for incongruent trials. The bottom row shows the mapping between stimuli and response hands

followed a $2 \times 2$ factorial design with factors of target color (red/green) and flanker color (red/green) counterbalanced and presented in random order across trials. This design ensured that the target and flanker colors were congruent on half of the trials and were incongruent on the remaining half, and that the correct answer was red on half of the trials. Every 100 trials participants were invited to take a break before continuing.

\section{Results}

Response time (RT) is defined as the time between the onset of stimuli and the response. For the main analyses, trials in which response times were excessively fast $(<200 \mathrm{~ms})$ or excessively slow ( $>2000 \mathrm{~ms}$ ) were not considered in analyses $(0.9 \%)$. However, the influence of this choice is explored in detail below.

Mean accuracy \& RT effects Although accuracy was high overall, it was significantly higher on congruent trials $(M=97 \%)$ than incongruent trials $(M=95 \%, t(46)=$ $6.11, p<.05, d=0.89$ ). Response time analyses included only trials with accurate target responses. Mean RTs were faster on congruent $(\mathrm{M}=473 \mathrm{~ms})$ than incongruent trials $(\mathrm{M}=494 \mathrm{~ms}, t(46)=10.20, p<.05, d=1.49)$.

Delta plots Delta plots were constructed by first computing the deciles $(0.1,0.2, \ldots, 0.9$ quantiles $)$ of response time distributions for the congruent and incongruent conditions for each participant. Decile values for the congruent condition were then subtracted from those for the incongruent condition, providing a measure of the congruency effect at each decile for each participant. Decile values were also averaged across the congruent and incongruent conditions, providing an average response time for each decile and each participant. To construct delta plots, these measures were averaged over participants, a procedure known as Vincentizing (Rouder \& Speckman, 2004), and the average effect for each decile was plotted against the average RT for that decile.

The delta plot for the color Eriksen task is shown in Fig. 4. The delta plot appears to be linear with positive slope, in line with previous findings that both the mean and standard deviation of response times are larger for the incongruent condition (Servant, Montagnini, \& Burle, 2014). An orthogonal-polynomial-contrast trend analysis (Grant, 1956) was used to quantify the shape of the delta plots. This approach was suggested by Burle et al. (2014) as being superior to other methods for characterizing the shape of delta plots, such as fitting regression models. For

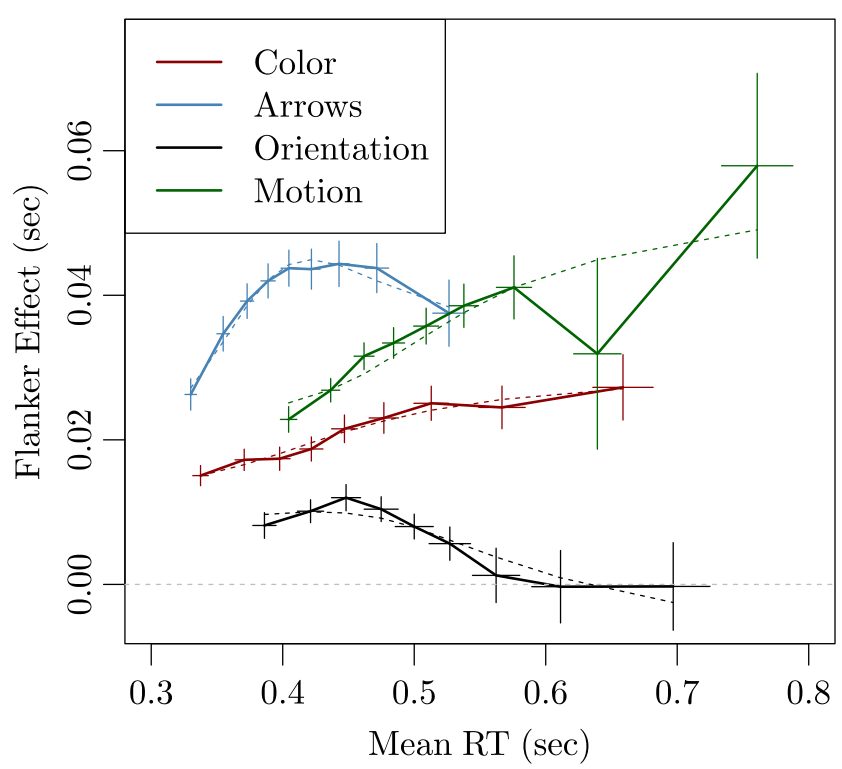

Fig. 4 Delta plots from Experiments 1-4. Solid lines denote delta plots for Experiment 1 (color), Experiment 2 (arrows), Experiment 3 (orientation) and Experiment 4 (motion). Error bars denote standard errors of the effect ( $y$-axis) and of the average RT ( $x$-axis). Dotted lines are estimates derived from grand mean parameters of the polynomial factor analysis 
the trend analysis, the flanker effect at each decile for each participant served as the dependent variable in a repeated measures ANOVA. The corresponding deciles were treated as factors, and orthogonal linear and quadratic contrasts were used to assess how the flanker effect varied with decile. The results suggest that the delta plot from Experiment 1 has a significant positive linear slope $(F(1,368)=45.51, p<$ $.05)$, but no quadratic trend $(F(1,368)=.001, p=.97)$. Whereas this analysis treats the mean RT deciles as equally spaced factors, for display purposes the predicted flanker effects from the fitted polynomial trend models at each decile are shown as a function of that decile's mean RT, as is typical when drawing delta plots. The predicted polynomial for the color flanker is shown as a dotted line in Fig. 4, and suggests that the estimated model provides a good fit to the data.

The number of delta-plot points In the main analyses, delta plots were constructed by using 9 deciles of the RT distributions. However, several other choices have been used in previous studies, and there is no correct choice. For example, a delta plot can be built with only four points by using quintiles $(.2, .4, .6$ and .8 percentiles). Unfortunately, however, this choice might affect the shape of the delta plot and the statistical results used to characterize it. Alternatives to using delta plots for characterizing interference effects have been proposed that are not sensitive to this choice (Gajdos, Servant, Hasbroucq, and Davranche, 2020), however, as our goal here is to investigate delta plot shapes we take a straightforward approach for ensuring that our results are not beholden to the number of delta plot points. Delta plots were constructed and trend analyses applied for several choices of how many quantiles were used to construct the plot. Figure 5a shows that for the

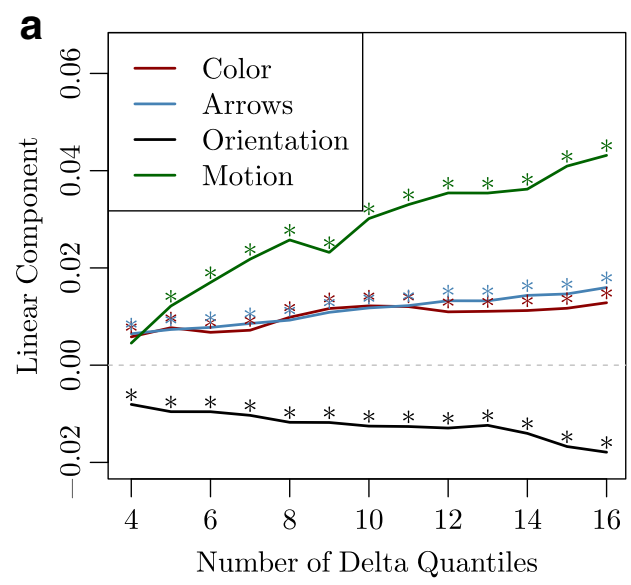

Fig. 5 The effect of the number of delta plot points on the shape of the delta function. Delta plots were constructed by using between four and 16 quantiles. For each choice, the linear (a) and quadratic (b) trends were measured for each experiment. Although the magnitude of the color Eriksen task of Experiment 1 the linear component is significantly greater than zero, and is of similar magnitude, regardless of how many points are used to build the delta plot. Likewise, the quadratic component (Fig. 5b) is never significantly different than zero. Taken together, these results suggest that the delta plot for a color version of the Eriksen flanker task is a positive-going straight line, regardless of how many quantiles are used to construct it.

The choice of RT cutoff Extremely slow responses clearly reflect processes other than those of interest in these simple conflict tasks, such as lapses of attention, or taking uninvited breaks. In the main analyses we consider responses slower than 2 seconds as reflecting such nuisance trials, and do not use them in constructing delta plots. This choice, however, is arbitrary and may have an impact on the shape of delta plots. In particular, the inclusion or exclusion of slow responses will primarily affect the slowest quantiles, which are critical for determining whether the delta plot is straight or curvilinear. The delta plot analysis was therefore conducted for a range of upper cut offs, and for each level of cut off the choice of how many points were used to construct the plot was also varied. Figure $6 \mathrm{a}$ shows whether the quadratic component was significant for each combination of upper RT cut off ( $x$-axis) and number of delta plot points ( $y$-axis). For the majority of choices the quadratic component is not significantly different than zero (indicated by white), as in the main analysis. However, for extremely short cut offs the delta plot exhibits some downward curvature (indicated by red). Although the flanker task is easy, removing responses that are slower than one second would clearly be removing trials that legitimately reflect the processes of interest, and would disproportionately affect the slower incongruent condition. We therefore suspect that

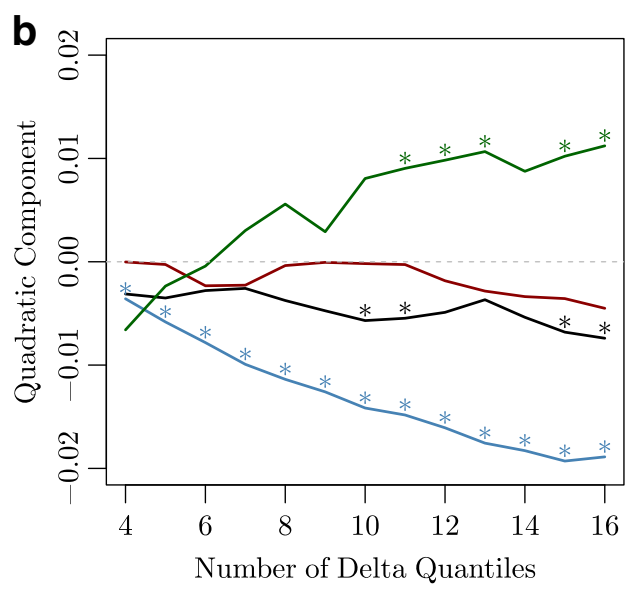

trends varies with the number of delta plot points in some experiments, the overall pattern and significance (denoted by stars) of the trends is largely consistent regardless of how many quantiles were used to construct the delta plot 

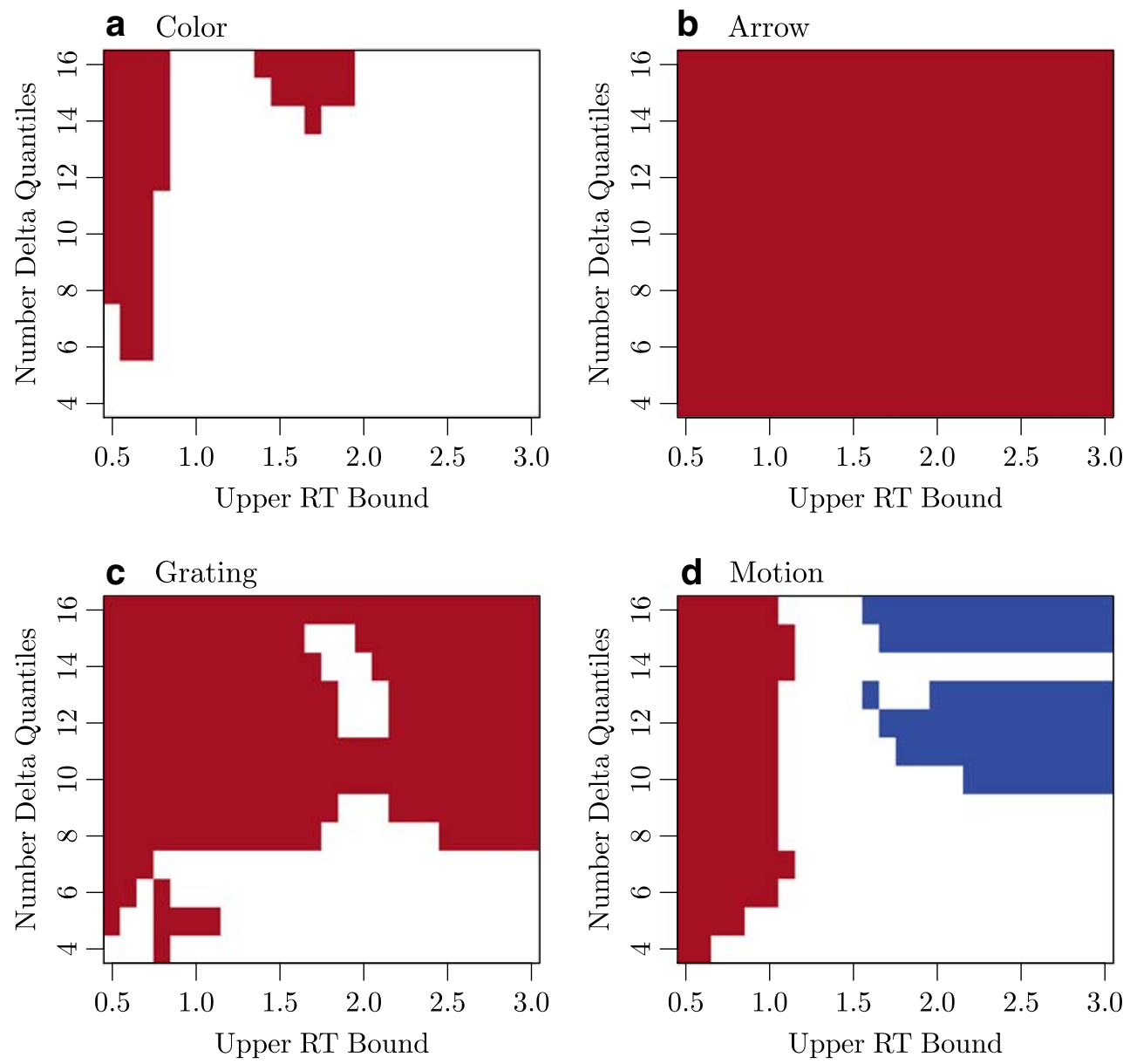

Fig. 6 The choice of upper RT cutoff can affect the delta plot shape. Each panel shows the results of one of the four experiments. For each combination of upper RT bound ( $x$-axis) and how many quantiles were used to construct the delta plot ( $y$-axis), color indicates whether the quadratic trend was significantly negative (red), significantly positive (blue), or did not differ significantly from zero (white). A negative quadratic component is clearly present for the arrow (b) and grating (c) flanker tasks regardless of upper RT cutoff or number of delta plot points. However, even the color (a) and motion (d) delta plots can be made to have an inverted-U shape if an unreasonably low RT cutoff is used

this downward curvature reflects an artifact of using an unreasonably fast lower bound, but shows that the delta plot shape can be substantially altered by such "researcher degrees of freedom" (Simmons, Nelson, \& Simonsohn, 2011).

\section{Discussion}

The results of Experiment 1 confirm that when the target and flanker information is color, the resulting delta plot is a positive-going straight line. This result can be accounted for by several theories. For example, Ulrich et al. (2015) found a positive-going delta plot in an Eriksen task with letters as targets and flankers, and suggest that this trajectory resulted from a relatively slow accumulation of flanker information relative the accumulation of target information, compared with the rapid rise and fall of spatial information in a Simon task. Presumably the relative accumulation of target and flanker letters is similar to that for target and flanker colors, such that the delta plots should be positive-going in both cases. In addition, because the interfering color information in Experiment 1 does not have a spatial location component, a left/right lateralization account of the Simon effect also anticipates a positive-going delta plot for the Eriksen task with color.

\section{Experiment 2: arrow direction}

Although the flanker effect is clearly positive-going when color serves as the target and flanker information, some theories predict that flanker effects using different stimulus types should exhibit downward going delta plots. For example, previous results suggest that delta plots may have an inverted-U shape when targets and flankers are arrows pointing to the left or right (e.g., Ridderinkhof et al. 2005). Arrows are thought to produce reflexive shifts of spatial attention toward the direction begin pointed to (Stevens 
et al., 2008; Tipples, 2002), such that the arrow flankers may produce interfering information that evokes processing of left/right spatial location. If it is the presence of spatial location information that causes the Simon effect delta plot to be negative-going (Wiegand \& Wascher, 2005; Pratte et al., 2010), then the delta plot for arrow flanker tasks should also be negative-going. However, previous studies did not conduct formal analyses to measure the shape of arrow-flanker delta plots, so in Experiment 2 we characterized the delta plot shape for an Eriksen flanker task in which leftward and rightward-pointing arrows served as targets and flankers.

\section{Method}

Participants Fifty-two students participated in Experiment 2. Data from four participants were not analyzed due to overall low accuracy on the task $(<90 \%)$.

Stimuli \& design Experiment 2 was identical to Experiment 1 with the following exceptions. Targets and flankers were white arrows pointing rightward or leftward (see Fig. 3b). The rectangular body of each arrow was $1^{\circ}$ in height and $1.5^{\circ}$ in length, and the triangular head was $.5^{\circ}$ in length such that the entire arrow subtended $2^{\circ}$. Targets were presented centrally (with overlaid fixation circle), and flankers were centered $\pm 3^{\circ}$ from the target center. Consequently, a $1^{\circ}$ gap separated targets from flankers. Participants identified the target arrow direction as leftward with their left hand or rightward with their right hand. Because color was no longer relevant to the task in Experiments 2, 3 or 4 , positive and negative accuracy feedback was given by changing the fixation point to green for $150 \mathrm{~ms}$, or red for $1000 \mathrm{~ms}$, respectively.

\section{Results}

Excessively fast or slow trials $(0.5 \%)$ were not considered in analyses. Accuracy was higher on congruent trials $(M=$ $98 \%)$ than incongruent trials $(M=93 \%, t(47)=$ $10.23, p<.05, d=1.48)$, and mean response times were faster on congruent $(M=404 \mathrm{~ms})$ than incongruent trials $(M=442 \mathrm{~ms}, t(47)=14.43, p<.05, d=2.08)$. The delta plot for the arrow flanker task is shown in Fig. 4. The polynomial trend analysis revealed a positive linear slope $(F(1,376)=43.56, p<.05)$ and a negative quadratic component $(F(1,376)=58.47, p<.05)$. The quadratic model provides a good fit to the data (dotted line in Fig. 4), and suggests that the arrow flanker effect initially increases, but then levels off and decreases at longer RTs producing an inverted-U shape.

Figure 5a shows that the linear component for the arrow flanker task is significantly positive regardless of how many points are used to construct the delta plot. Likewise, Fig. 5b shows that the quadratic component is significantly negative regardless of this choice. The magnitude of the quadratic component does, however, appear to increase as more points are used to construct the delta plot. Such negative quadratic trends have been crucial for theory development, and this result shows that arbitrary analysis choices can substantially influence their magnitude. In addition to the arbitrary choice of how many quantiles are used to construct the delta plot, researches must decide whether some response times are too long to reasonably reflect the process of interest. However, Fig. $6 \mathrm{~b}$ shows that the quadratic component of the arrow flanker delta plot is significantly negative for a wide range of RT cut offs and number of delta plot quantiles tested.

\section{Discussion}

The results of Experiment 2 suggest that when leftward/rightward pointing arrows serve as target and flanker stimuli, the delta plot does not increase linearly, but instead has a clear inverted-U shape. Taken together with Experiment 1 , these results show that the delta plot shape for flanker tasks can be manipulated by altering the stimulus materials. This result is anticipated by the idea that delta plot shapes are determined by whether or not the distracting information involves a spatial component. The color stimuli used in Experiment 1 have no spatial component, and the delta plot is positive-going. Alternatively, the arrows in Experiment 2 cause leftward and rightward shifts in spatial attention, and the resulting delta plot exhibits an invertedU shape. Such non-monotonic delta plots are often taken to suggest that the irrelevant information is being actively suppressed over time (Ridderinkhof, 2002b), and it may be that such an automatic suppression process only occurs for stimuli that induce left/right spatial activation (Wiegand \& Wascher, 2005; Pratte et al., 2010).

\section{Experiment 3: orientation}

There are good reasons to suspect that spatial location might lead to qualitatively different interference effects than other stimulus types, such as the existence of leftright lateralized visual representations and motor mappings throughout the brain. However, it is also possible that other characteristics of the color and arrow stimuli determined the delta plot shapes in Experiments 1 and 2. For example, color information is processed by mid-level brain areas (McKeefry \& Zeki, 1997), and may even produce interference at the much higher level of semantic processing (Luo, 1999). Alternatively, spatial location is represented throughout the early visual cortex (Deyoe et al., 1996), and arrow direction automatically evokes shifts in spatial 
attention (Stevens et al., 2008) which also manifest in early visual areas (Somers, Dale, Seiffert, \& Tootell, 1999). Therefore, it is possible that the different delta plot shapes observed across Experiments 1 and 2 reflect how early in the visual processing stream the interfering information is processed, rather than the presence or absence of interfering spatial location information per se. In particular, rather than an automatic suppression process occurring only for interfering spatial information, suppression may occur for any interfering low-level visual information. This level-ofprocessing account would explain why high-level features such as letters and colors produce positive-going delta plots, whereas low-level features such as arrow direction in the flanker task and spatial location in the Simon task produce delta plots with negative-going components.

To differentiate between the spatial-location and level-ofprocessing explanations of delta plot shapes, Experiment 3 was a flanker task in which orientation served as the target and flanker feature dimensions. Like spatial location, orientation is a low-level visual feature that is represented throughout the early human visual system (Kamitani \& Tong, 2005). However, orientation should not evoke spatial location processing or cause shifts in spatial attention in the way that arrows do. Therefore, if negative-going delta plots only occur when spatial location serves as the interfering information, then the orientation flanker task should produce positive-going delta plots. Alternatively, if negative-going deltas result from interfering information that is processed early in the visual processing stream, then the orientation flanker delta should be negative-going.

\section{Method}

Participants Fifty-two students participated in Experiment 3. Data from three participants were not analyzed due to overall low accuracy on the task $(<90 \%)$.

Stimuli \& design Experiment 3 was similar to Experiment 1 with the following exceptions. Targets and flankers were 2D sine wave gratings (Fig. 3c). Gratings oscillated in luminance around the background gray level with $90 \%$ contrast and 1.5 cycles / deg spatial frequency. Phases were selected randomly for each grating on each trial. Gratings subtended $4^{\circ}$ in diameter, and flanking gratings were centered $\pm 4.5^{\circ}$ from the target center, leaving a minimum $0.5^{\circ}$ gap between the target and flanker boundaries. Target gratings were oriented $\pm 45^{\circ}$ around vertical. Flanker orientations were also $\pm 45^{\circ}$, and could additionally be vertically oriented $\left(0^{\circ}\right)$ providing a neutral-context condition (although data from this condition are not analyzed). Experiment 3 was a $2 \times 3$ factorial design with two target orientation conditions $\left(-45^{\circ}\right.$ and $\left.45^{\circ}\right)$ and three flanker orientation conditions $\left(-45^{\circ}, 0^{\circ}\right.$ and $\left.45^{\circ}\right)$ counterbalanced across the experiment. This design provided 300 congruent and 300 incongruent trials for each participant.

\section{Results}

Excessively fast or slow trials $(1.0 \%)$ were not considered in analyses. Accuracy was marginally higher on congruent trials $(M=96 \%)$ than incongruent trials $(M=$ $95 \%, t(48)=3.07, p<.05, d=0.44)$, and mean response times were faster on congruent $(M=528 \mathrm{~ms})$ than incongruent trials $(M=533 \mathrm{~ms}, t(48)=2.30, p<$ $.05, d=0.33)$. Whereas the effect in mean RT is small, the delta plot (Fig. 4) shows a clear congruency effect that is largest for fast responses and decreases with time. The polynomial trend analysis suggests a significant negative linear component $(F(1,384)=20.51, p<.05)$ and a negative quadratic component that is marginally significant $(F(1,384)=3.32, p=.07)$.

The negative-going linear component is significant and of similar magnitude regardless of how many percentiles are used to construct the delta plot (Fig. 5a). Although the quadratic component is also negative for all choices of percentile (Fig. 5b), this inverted-U shape is only significant for a few of these choices. However, Fig. $6 c$ shows that although the negative quadratic component is not highly stable when the upper RT cut off is 2 seconds, as was used in the main analysis, for many other choices of this bound the negative quadratic trend is significant, and this pattern holds for many choices of how many percentiles are used to construct the delta plot. Overall, the delta plot of an Eriksen flanker task that utilizes oriented gratings is clearly downward going, and shows some evidence that this negative trajectory follows an inverted-U shape rather than being purely linear.

\section{Discussion}

If downward-going delta plots such as in the Simon task and Eriksen flanker task with arrows are caused by the presence of interfering spatial location information, then a flanker task with oriented grating stimuli should produce a positivegoing delta. However, the delta plot from Experiment 3 is clearly negative-going, with a shape that is similar to that of the typical Simon effect delta plot. This negative-going delta plot for the orientation flanker task is not easily explained by the idea that negative-going delta plots reflect the presence of spatial location as the interfering feature dimension, as oriented gratings presumably do not evoke spatial location processing. However, the results from Experiments 1-3 are concordant with a level-of-processing account, by which delta plots should have a negative-going component if the 
interfering dimension is a low-level visual feature, such as spatial location, arrow direction, or orientation, whereas higher-level interfering information such as colors or letters should produce positive-going deltas.

\section{Experiment 4: motion}

The oriented gratings in Experiment 3 were assumed to be low-level visual stimuli that do not convey location information. However, it is difficult to completely rule out the possibility that gratings, or any other stimulus, conveys some spatial location signal. For example, in Experiment 3 it is possible that spatial information was inferred from the grating patterns, such as the $45^{\circ}$ grating being interpreted as "up and to the right". Consequently, the negative-going delta plot for orientation does not provide strong evidence against the spatial-location account of negative-going delta plots, without somehow ruling out the possibility that spatial information was activated by the gratings. In Experiment 4 we therefore sought to examine stimuli that do evoke a spatial response, but also require higher levels of processing. If spatial location is the critical feature, then these delta plots should be negative-going. Alternatively, if it is the level of processing that determines delta plot shapes, then these stimuli should produce positive-going deltas.

Experiment 4 was an Eriksen flanker task in which global motion direction served as the target and flanker information. Motion has been shown to produce flanker interference effects in mean RT (Lange-Malecki \& Treue, 2012), but to our knowledge delta plots of this task have not been examined. The target and flankers were fields of dots that, on average, moved leftward or rightward (Fig. 3d). Critically, each dot was only presented for $100 \mathrm{~ms}$ before it disappeared and re-appeared at a new location within the stimulus, and its motion direction was perturbed by noise such that no one dot moved exactly leftward or rightward. Consequently, the overall leftward and rightward motion energy was global, and must be constructed by the perceptual system by somehow integrating the local dot motions across space and time (Burr \& Santoro, 2001). Such global motion perception cannot be accomplished by early visual areas, but occurs within regions located in the medial temporal lobe (Newsome \& Pare, 1988; Tootell et al., 1995; Rees, Friston, \& Koch, 2000). This motion stimulus is therefore a higher-level visual feature like color, and according to the level-of-processing account should produce a positive-going delta plot. Alternatively, such motion information has been shown to automatically evoke spatial processing. For example, irrelevant motion toward the left or right causes similar effects as irrelevant spatial location does in a Simon task, suggesting that such motion automatically activates left/right spatial responses
(Bosbach, Prinz, \& Kerzel, 2004). Therefore, whereas the level-of-processing account predicts a positive-going delta plot, if the presence of spatial information is what determines the delta plot shape, then delta plots should be negative-going for a flanker task in which motion direction serves as the stimulus dimension.

\section{Method}

Participants 59 students participated in Experiment 4. Data from three participants were not analyzed due to overall low accuracy on the task $(<90 \%)$.

Stimuli \& design Experiment 4 was similar to Experiment 1 with the following exceptions. Target and flanker stimuli were fields of 300 moving dots, masked by a circular aperture subtending $3^{\circ}$ (Fig. 3d). Flanker apertures were centered at $\pm 3.5^{\circ}$ from the target center, leaving at least a $0.5^{\circ}$ gap between stimuli. Dots were small white squares ( $4 \times 4$ pixels) with missing corner pixels, making them appear as round dots at normal viewing distances. Dots were initially located in random positions within the stimulus aperture, and moved at a speed of $3 \mathrm{deg} / \mathrm{sec}$ for one second before the response period. Dots had a limited lifetime, such that a dot would disappear every $100 \mathrm{~ms}$ and re-appear at a new, randomly chosen location within the stimulus aperture. The initial age of each dot was random, such that dots disappeared at independent times. The heading direction of each dot was either leftward or rightward, perturbed by adding a small amount of Gaussian noise $(\sigma=0.01$ degrees visual angle) to the $\mathrm{x}$-and $\mathrm{y}$-positions on each frame (60 Hz display). The dots therefore exhibited Brownian motion, with either a leftward or rightward average motion direction. The left/right motion directions of the target and flankers were counterbalanced in a $2 \times 2$ factorial design.

\section{Results}

Excessively fast or slow trials $(0.4 \%)$ were not considered in analyses. Accuracy was higher on congruent trials $(M=$ $98 \%)$ than incongruent trials $(M=95 \%, t(55)=$ 8.13, $p<.05, d=1.09$ ), and mean response times were faster on congruent $(M=535 \mathrm{~ms})$ than incongruent trials $(M=573 \mathrm{~ms}, t(55)=9.90, p<.05, d=1.32)$. Figure 4 shows the delta plot for the motion flanker task. Although the plot appears to decrease at the 0.8 percentile, the large error bars for the slowest percentiles suggest that such patterns may not be reliable. Indeed, the polynomial trend analysis indicates a significant linear component with positive slope $(F(1,440)=16.99, p<.05)$, but no evidence for a quadratic trend $(F(1,440)=0.27, p=$ .61). This delta plot is therefore most similar to the linearly 
increasing function observed in the color flanker task of Experiment 1.

The linear trend is significantly positive regardless of how many points are used to construct the delta plot (Fig. 5a), whereas the quadratic component is not significant for most choices (Fig. 5b). When more than 10 points were used to construct the delta plot the trend analysis does indicate a significant quadratic trend, but it is positive, implying a non-linear increase in the delta plot rather than a leveling-off as is seen for arrow stimuli. This pattern in the quadratic component is also stable for a range of choices for the upper RT cut off (Fig. 6d). However, as with the color flanker task, using an upper bound on RT of one second or less causes a significant negative-going quadratic component for the motion flanker. Again, this result is likely an artifact of removing trials more aggressively in the slower incongruent condition than the congruent condition, and highlights that care must be taken with making these arbitrary choices in analysis.

\section{General discussion}

Distributional analyses have revealed substantial differences across conflict tasks such as Stroop and Simon (e.g., Pratte et al. 2010). These results have led to several theories of why the time course of interference differs across tasks. However, these tasks differ from one another in many aspects, making it difficult to isolate any particular cause for the different patterns of behavioral data across them. We therefore assessed whether the delta plot shape can be manipulated within an Eriksen flanker task by systematically varying the stimulus materials, while keeping other aspects of the task constant. Delta plots were positivegoing when the relevant stimulus feature was color or motion, but were inverted-U-shaped and negative-going when stimuli were arrows or oriented gratings, respectively. These results show that the delta plot shape does not only change across tasks, but can be affected by manipulating stimulus characteristics within otherwise identical tasks.

Examining how particular manipulations affect the delta plot shape provides a powerful way to assess theories of why delta plots are sometimes positive-going functions and sometimes negative. For example, we and other researchers have suggested that the downward-going delta plots observed for Simon effects might reflect the fact that spatial location serves as the interfering information in a Simon task (Wiegand \& Wascher, 2005; Pratte et al., 2010). Some of the flanker results here are consistent with this proposal, such as the inverted-U-shaped delta plot when leftwardand rightward-pointing arrows served as targets and flankers (Experiment 2). However, other results undermine this explanation. Whereas oriented gratings should not evoke spatial location processing, the delta plot for a flanker task using orientation as the target and flankers is clearly negative-going (Experiment 3). Moreover, whereas leftward and rightward motion stimuli in Experiment 4 should evoke spatial location processing, the delta plot for this task is clearly positive-going. Taken together, it seems that the mere presence or absence of spatial location information in the interfering stimulus dimension is not sufficient to explain why some tasks exhibit negative delta plot slopes.

Comparing delta plots across the four flanker tasks (Fig. 4) rules out several other possible aspects of conflict tasks that may be thought to drive the shape of delta plots. For example, the extent to which the delta plots have a negative-going component does not appear to depend on the overall response speed ( $x$-axis), nor on the overall effect size ( $y$-axis). Task difficulty also does not appear to drive the delta plot shape, as accuracy in all four flanker tasks was highly similar, as were the small but consistent congruency effects on accuracy. Finally, the target-to-flanker distance was not constant across all experiments, and flanker effects in mean RT have been shown to be larger when the flankers are closer to the target (Eriksen \& Eriksen, 1974). However, the colored boxes in Experiment 1 had the same height, total length, and target-to-flanker spacing as the arrows in Experiment 2, yet the delta plots were markedly different for these tasks. It is difficult to directly compare stimulus sizes and spacing across experiments here, since the stimuli have different shapes. However, stimulus size and target-toflanker spacing is one of many stimulus variables that could be parametrically manipulated in a flanker task to further explore what might cause delta plots to change shape.

The only consistent pattern we observed across experiments is that flanker delta plots have a negative-going component when the interfering information is processed early in the visual cortex, including spatial location and orientation, and are positive-going for higher-level interfering information such as letters, color, and global motion direction. There are several aspects of the stimuli that might cause such a level-of-processing effect, and many formal frameworks that might describe why it exists. For example, perhaps low-level information can be processed more independently of later processing stages such as decision making. Schwarz and Miller (2012) showed that a model with two serial processing stages, such as a perceptual analysis stage and subsequent motor preparation stage, can produce a negative-going delta plot if the correlation between stage completion times varies across congruent and incongruent conditions. If these correlations also depend on the degree to which the stages are independent of one another across stimulus materials, then delta plot shapes would depend on the level of processing of the target and flanker stimuli. This account is clearly speculative, especially as Schwarz and Miller (2012) demonstrated that several formal models can 
account for a range of delta plot shapes. However, if such models make predictions regarding what factors should and should not affect the delta plot shape, then measuring delta plots across within-task manipulations may be useful for testing them.

Ulrich et al. (2015) suggested that the different delta plot shapes for Simon and Eriksen tasks reflect a difference in how quickly the irrelevant information accumulates relative to the target information. In the Simon task the interfering spatial information is thought to accumulate and then dissipate rapidly, thereby affecting only fast responses and consequently producing a negative-going delta plot. Alternatively, the positive-going flanker delta plot implies that the flanker information accumulates more slowly relative to target information, such that only slow responses exhibit a congruency effect. For this account to explain the flanker results reported here, the relative time course of processing target and flanker information would have to differ across stimulus types. For example, it is possible that for low-level stimuli the two flankers combine efficiently, such that together the flanker information accumulates faster than information from the single target, leading to negative-going delta plots. If higher-level stimuli do not enjoy such a boost from aggregating over the two flankers, then in experiments using higher-level stimuli the centrally presented target might accumulate faster than the flankers, producing a positive-going delta plot. There is evidence for such a gain across redundant stimuli, such as when multiple copies of the same target letter are presented simultaneously (e.g., Eriksen and Lappin 1965; Fournier \& Eriksen 1990), or when two lines serve as targets in a detection task rather than just one (Schwarz \& Ischebeck, 1994). We do not know of any evidence that the magnitude of this redundancy gain varies across stimulus types, or whether in the Eriksen task any benefit of aggregating two flankers can overcome the advantage of processing the foveated and attended target. Clearly more work is needed to understand how targets and flankers might interact, and how the consequences affect delta plot shapes. Fortunately, the Eriksen flanker task provides an extraordinary amount of flexibility for addressing such questions, such as by varying the number of targets and flankers, the distance between targets and flanker, and the degree to which target and flanker information overlap.

Whereas the models considered above were designed to account for conflict effects in general (e.g., Schwarz \& Miller 2012; Ulrich et al. 2015), several other theories have been proposed to account for the Eriksen flanker effect in particular. In line with the original purpose of the flanker task developed by Eriksen and Eriksen (1974), many of these theories suggest that flanker effects are the result of attention spilling over to the flanker stimuli. Such theories have enjoyed substantial development since the work of
Eriksen \& Eriksen, including variants that have been built into formal models (e.g., Logan, 1996; Hübner, Steinhauser, \& Lehle, 2010). For example, White, Ratcliff, and Starns (2011) proposed the shrinking spotlight model based on the idea that attention acts like a zoom lens (Eriksen \& St. James, 1986). According to this model, spatial attention is diffuse at the beginning of a flanker trial and, to some degree, overlaps with the flankers such that they will affect behavior. But over time attention becomes more focused on the target, such that the flankers have less influence. This model accounts for the often-observed finding that incorrect responses in the incongruent condition of a flanker task tend to be very fast (Gratton, Coles, Sirevaag, Eriksen, \& Donchin, 1988), implying that the flanker compatibility effect in accuracy is initially large, but quickly diminishes as evidenced by equivalent accuracy for congruent and incongruent trials that have slow RTs (White et al., 2011). However, this model predicts positive-going delta plots (Servant et al., 2014), and so cannot account for the arrow and orientation flanker effects observed here. We did not examine effects in accuracy here given that the tasks were trivially easy and consequently accuracy was very high in all conditions. However, future work that is designed to examine both response time and accuracy may help to shed light on why the compatibility effects differ for different stimulus materials, and more generally, how the delta plot shape might be related to effects in accuracy.

The results across Experiments 1-4 rule out some factors as necessary determinants of delta plot shape, such as spatial location. However, other manipulations have been shown to alter the shape of delta plots, suggesting that there may be several task characteristics that can do so. For example, Hübner and Töbel (2019) showed that the flanker delta plot can be made to be negative-going if the flankers are presented prior to the target, suggesting that target-distractor timing can play a role (see also Mattler 2003). Simon tasks exhibit negative-going delta plots when responses and stimuli are horizontally aligned, but are positive-going if the stimuli and responses are vertically aligned (Pratte et al., 2010; Wiegand \& Wascher, 2005), implying that spatial location may also affect the delta plot shape. Burle et al. (2014) examined a flanker task with letters, and observed the typical positive-going delta plot when the data were averaged over trials. However, they also measured muscle activity at the hands, and when considering only those trials on which a small amount of muscle activation was observed at the incorrect-response hand, the flanker delta plot had a negative-going component (see also Eriksen, Coles, Morris, \& O'hara, 1985). This result was taken to suggest that whatever process makes delta plots negative-going in the Simon task is also present in the Eriksen task, just to a lesser degree. These results are all consistent with the general idea proposed by Ridderinkhof (2002a) and Ridderinkhof 
(2002b) that any negative-going component in delta plots reflects an inhibition of the irrelevant information. However, it is not clear why such inhibition would be high in the Simon task and the Eriksen flanker tasks using orientation or arrow direction, but low in the Stroop task and Eriksen tasks where color, motion direction, or letters serve as stimuli.

More work is needed to understand why some conflict tasks produce negative-going delta plots and some do not. It may be the case that interesting aspects of conflict processing such as information suppression drive delta plot shapes (Ridderinkhof, 2002a). Or more generally, they may reflect aspects of how target and distractor processing differs across tasks (Ulrich et al., 2015). However, it is also possible that delta plot shapes are uninteresting, such as reflecting statistical properties of response time distributions that are not necessarily coupled with aspects of the cognitive architecture (Zhang \& Kornblum, 1997). Fortunately, the collection of conflict tasks including Stroop, Simon and Eriksen allow for remarkable flexibility in implementation despite their simplicity, and combining these experimental approaches with contemporary models of response time distributions may prove to be highly productive for understanding the nature of conflict resolution.

Acknowledgements Many thanks to Elizabeth Beene, Abigail Phelps, and Jayden Pendegraph for assistance with data collection; to Corey White, Mathieu Servant, and two anonymous reviewers for their invaluable feedback; and to researchers like Charles Eriksen, whose work demonstrates that some of the most important scientific discoveries can come from the simplest experiments.

Open practices statement The data for all experiments are available on the author's website or by contacting the author.

\section{References}

Bosbach, S., Prinz, W., \& Kerzel, D. (2004). A Simon effect with stationary moving stimuli. Journal of Experimental Psychology: Human Perception and Performance, 30(1), 39-55. https://doi.org/10.1037/0096-1523.30.1.39

Brainard, D. H. (1997). The psychophysics toolbox. Spatial Vision, 10(4), 433-436. https://doi.org/10.1163/156856897X00357

Burle, B., Spieser, L., Servant, M., \& Hasbroucq, T. (2014). Distributional reaction time properties in the Eriksen task: Marked differences or hidden similarities with the Simon task? Psychonomic Bulletin and Review, 21(4), 1003-1010. https://doi.org/10.3758/ s13423-013-0561-6

Burr, D. C., \& Santoro, L. (2001). Temporal integration of optic flow, measured by contrast and coherence thresholds. Vision Research, 41(15), 1891-1899. https://doi.org/10.1016/S0042-6989(01)0007 $2-4$

Davranche, K., Hall, B., \& McMorris, T. (2009). Effect of acute exercise on cognitive control required during an Eriksen flanker task. Journal of Sport and Exercise Psychology, 31(5), 628-639. https://doi.org/10.1123/jsep.31.5.62810.1123/jsep.31.5.628
De Jong, R., Liang, C. C., \& Lauber, E. (1994). Conditional and unconditional automaticity: a dual-process model of effects of spatial stimulus-response correspondence. Journal of Experimental Psychology:, Human Perception and Performance, 20(4), 731-750. https://doi.org/10.1037//0096-1523.20.4.731

Deyoe, E. A., Carman, G. J., Bandettini, P., Glickman, S., Wieser, J., Cox, R., ..., Neitz, J. (1996). Mapping striate and extrastriate visual areas in human cerebral cortex. Proceedings of the National Academy of Sciences of the United States of America, 93(6), 2382-2386. https://doi.org/10.1073/pnas.93.6.2382

Eaton, J. W., Bateman, D., Hauberg, S., \& Wehbring, R. (2014). GNU Octave version 3.8.1 manual: a high-level interactive language for numerical computations. Scotts Valley: CreateSpace Independent Publishing Platform.

Eriksen, B. A., \& Eriksen, C. W. (1974). Effects of noise letters upon the identification of a target letter in a nonsearch task. Perception and Psychophysics, 16(1), 143-149. https://doi.org/10.3758/BF0 3203267

Eriksen, C. W. (1995). The flankers task and response competition: a useful tool for investigating a variety of cognitive problems. In Bundesen, C., \& Shibuya, H. (Eds.) Visual cognition: visual selective attention. https://doi.org/10.1080/13506289508401726 (Vol. 2 and 3, pp. 101-118): Taylor and Francis Group.

Eriksen, C. W., Coles, M. G., Morris, L. R., \& O'hara, W. P. (1985). An electromyographic examination of response competition. Bulletin of the Psychonomic Society, 23(3), 165-168. https://doi.org/10.3758/BF03329816

Eriksen, C. W., \& Lappin, J. S. (1965). Internal perceptual system noise and redundancy in simultaneous inputs in form identification. Psychonomic Science, 2(1), 351-352. https://doi.org/10. 3758/BF03343493

Eriksen, C. W., \& St. James, J. D. (1986). Visual attention within and around the field of focal attention: A zoom lens model. Perception and Psychophysics, 40(4), 225-240. https://doi.org/10.3758/BF0 3211502

Fenske, M. J., \& Eastwood, J. D. (2003). Modulation of focused attention by faces expressing emotion: evidence from flanker tasks. Emotion, 3(4), 327-343. https://doi.org/10.1037/1528-3542.3. 4.327

Fournier, L. R., \& Eriksen, C. W. (1990). Coactivation in the perception of redundant targets. Journal of Experimental Psychology: Human Perception and Performance, 16(3), 538-550. https://doi.org/10.1037/0096-1523.16.3.538

Gajdos, T., Servant, M., Hasbroucq, T., \& Davranche, K. (2020). A measure of the interference effect distribution. Behavior Research Methods, 52(4), 1629-1639. https://doi.org/10.3758/s13428-01901339-6

Grant, D. A. (1956). Analysis-of-variance tests in the analysis and comparison of curves. Psychological Bulletin, 53(2), 141-154. https://doi.org/10.1037/h0038479

Gratton, G., Coles, M. G., Sirevaag, E. J., Eriksen, C. W., \& Donchin, E. (1988). Pre- and poststimulus activation of response channels: a psychophysiological analysis. Journal of Experimental Psychology: Human Perception and Performance, 14(3), 331-344. https://doi.org/10.1037/0096-1523.14.3.331

Hommel, B. (1993). The relationship between stimulus processing and response selection in the Simon task: Evidence for a temporal overlap. Psychological Research, 55(4), 280-290. https://doi.org/10.1007/BF00419688

Hübner, R., Steinhauser, M., \& Lehle, C. (2010). A dual-stage twophase model of selective attention. Psychological Review, 117(3), 759-784. https://doi.org/10.1037/a0019471

Hübner, R., \& Töbel, L. (2019). Conflict resolution in the Eriksen flanker task: Similarities and differences to the Simon task. PLoS ONE 14(3). https://doi.org/10.1371/journal.pone.0214203 
Kamitani, Y., \& Tong, F. (2005). Decoding the visual and subjective contents of the human brain. Nature Neuroscience, 8(5), 679-685. https://doi.org/10.1038/nn1444

Kleiner, M., Brainard, D. H., Pelli, D. G., Broussard, C., Wolf, T., \& Niehorster, D. (2007). What's new in Psychtoolbox-3? Perception, 36(14), 1-16. https://doi.org/10.1068/v070821

Kopp, B., Rist, F., \& Mattler, U. (1996). N200 in the flanker task as a neurobehavioral tool for investigating executive control. Psychophysiology, 33(3), 282-294. https://doi.org/10.1111/j.14698986.1996.tb00425.x

Kornblum, S., Hasbroucq, T., \& Osman, A. (1990). Dimensional overlap: cognitive basis for Stimulus-Response CompatibilityA model and taxonomy. Psychological Review, 97(2), 253-270. https://doi.org/10.1037/0033-295X.97.2.253

Lange-Malecki, B., \& Treue, S. (2012). A flanker effect for moving visual stimuli. Vision Research, 62, 134-138. https://doi.org/10. 1016/j.visres.2012.03.016

Logan, G. D. (1996). The CODE theory of visual attention: an integration of space-based and object-based attention. Psychological Review, 103(4), 603-649. https://doi.org/10.1037/0033-295X. 103.4.603

Luo, C. R. (1999). Semantic competition as the basis of Stroop interference: Evidence from color-word matching tasks. Psychological Science, 10(1), 35-40. https://doi.org/10.1111/1467-9280.00103

Mattler, U. (2003). Delayed flanker effects on lateralized readiness potentials. Experimental Brain Research, 151, 272-288. https://doi.org/10.1007/s00221-003-1486-5

McKeefry, D. J., \& Zeki, S. (1997). The position and topography of the human colour centre as revealed by functional magnetic resonance imaging. Brain: A Journal of Neurology, 120(12), 2229-2242. https://doi.org/10.1093/brain/120.12.2229

Newsome, W. T., \& Pare, E. B. (1988). A selective impairment of motion perception following lesions of the middle temporal visual area (MT). Journal of Neuroscience, 8(6), 2201-2211. https://doi.org/10.1523/jneurosci.08-06-02201.1988

Pelli, D. G. (1997). The VideoToolbox software for visual psychophysics: transforming numbers into movies. Spatial Vision, 10(4), 437-442. https://doi.org/10.1163/156856897X00366

Pratte, M. S., Rouder, J. N., Morey, R. D., \& Feng, C. (2010). Exploring the differences in distributional properties between Stroop and Simon effects using delta plots. Attention, Perception, and Psychophysics, 72(7), 2013-2025. https://doi.org/10.3758/APP.72. 7.2013

Rafal, R., Gershberg, F., Egly, R., Ivry, R., Kingstone, A., \& Ro, T. (1996). Response channel activation and the lateral prefrontal cortex. Neuropsychologia, 34(12), 1197-1202. https://doi.org/10. 1016/0028-3932(96)00045-0

Rees, G., Friston, K., \& Koch, C. (2000). A direct quantitative relationship between the functional properties of human and macaque v5. Nature Neuroscience, 3(7), 716-723. https://doi.org/10.1038/76673

Ridderinkhof, K. R. (2002a). Activation and suppression in conflict tasks: empirical clarification through distributional analyses. In Prinz, W., \& Hommel, B. (Eds.) Common mechanisms in perception and action: Attention and performance xix, (pp. 494519). Oxford: Oxford University Press.

Ridderinkhof, K. R. (2002b). Micro- and macro-adjustments of task set: Activation and suppression in conflict tasks. Psychological Research, 66(4), 312-323. https://doi.org/10.1007/s00426002-0104-7

Ridderinkhof, K. R., Scheres, A., Oosterlaan, J., \& Sergeant, J. A. (2005). Delta plots in the study of individual differences: New tools reveal response inhibition deficits in $\mathrm{AD} / \mathrm{HD}$ that are eliminated by methylphenidate treatment. Journal of Abnormal
Psychology, 114(2), 197-215. https://doi.org/10.1037/0021-843X. 114.2.197

Rouder, J. N., \& Speckman, P. L. (2004). An evaluation of the Vincentizing method of forming group-level response time distributions. Psychonomic Bulletin and Review, 11(3), 419-427. https://doi.org/10.3758/BF03196589

Schwarz, W., \& Ischebeck, A. (1994). Coactivation and statistical facilitation in the detection of lines. Perception, 23(2), 157-168. https://doi.org/10.1068/p230157

Schwarz, W., \& Miller, J. (2012). Response time models of delta plots with negative-going slopes. Psychonomic Bulletin and Review, 19(4), 555-574. https://doi.org/10.3758/s13423-012-0254-6

Servant, M., Montagnini, A., \& Burle, B. (2014). Conflict tasks and the diffusion framework: Insight in model constraints based on psychological laws. Cognitive Psychology, 72, 162-195. https://doi.org/10.1016/j.cogpsych.2014.03.002

Simmons, J. P., Nelson, L. D., \& Simonsohn, U. (2011). False-positive psychology: Undisclosed flexibility in data collection and analysis allows presenting anything as significant. Psychological Science, 22(11), 1359-1366. https://doi.org/10.1177/0956797611417632

Simon, J. R. (1969). Reactions toward the source of stimulation. Journal of Experimental Psychology, 81(1), 174-176. https://doi.org/ $10.1037 / \mathrm{h} 0027448$

Somers, D. C., Dale, A. M., Seiffert, A. E., \& Tootell, R. B. (1999). Functional MRI reveals spatially specific attentional modulation in human primary visual cortex. Proceedings of the National Academy of Sciences of the United States of America, 96(4), 1663-1668. https://doi.org/10.1073/pnas.96.4.1663

Speckman, P. L., Rouder, J. N., Morey, R. D., \& Pratte, M. S. (2008). Delta plots and coherent distribution ordering. American Statistician, 62(3), 262-266. https://doi.org/10.1198/000313008X333493

Stevens, S. A., West, G. L., Al-Aidroos, N., Weger, U. W., \& Pratt, J. (2008). Testing whether gaze cues and arrow cues produce reflexive or volitional shifts of attention. Psychonomic Bulletin and Review, 15(6), 1148-1153. https://doi.org/10.3758/PBR.15.6. 1148

Strasburger, H., Rentschler, I., \& Jüttner, M. (2011). Peripheral vision and pattern recognition: A review. Journal of Vision, 11(5), 13. https://doi.org/10.1167/11.5.13

Stroop, J. R. (1935). Studies of interference in serial verbal reactions. Journal of Experimental Psychology, 18(6), 643-662. https://doi.org/10.1037/h0054651

Thornton, I. M., \& Vuong, Q. C. (2004). Incidental processing of biological motion. Current Biology, 14(12), 1084-1089. https://doi.org/10.1016/j.cub.2004.06.025

Tipples, J. (2002). Eye gaze is not unique: Automatic orienting in response to uninformative arrows. Psychonomic Bulletin and Review, 9(2), 314-318. https://doi.org/10.3758/BF03196287

To, L., Woods, R. L., Goldstein, R. B., \& Peli, E. (2013). Psychophysical contrast calibration. Vision Research, 90, 15-24. https://doi.org/10.1016/j.visres.2013.04.011

Tootell, R. B., Reppas, J. B., Kwong, K. K., Malach, R., Born, R. T., Brady, T. J., ..., Belliveau, J. W. (1995). Functional analysis of human MT and related visual cortical areas using magnetic resonance imaging. Journal of Neuroscience, 15(4), 3215-3230. https://doi.org/10.1523/jneurosci.15-04-03215.1995

Ulrich, R., Schröter, H., Leuthold, H., \& Birngruber, T. (2015). Automatic and controlled stimulus processing in conflict tasks: Superimposed diffusion processes and delta functions. Cognitive Psychology, 78, 148-174. https://doi.org/10.1016/j.cogpsych. 2015.02.005

Wagenmakers, E. J., \& Brown, S. (2007). On the linear relation between the mean and the standard deviation of a response time distribution. Psychological Review, 114(3), 830841. https://doi.org/10.1037/0033-295X.114.3.830 
White, C. N., Ratcliff, R., \& Starns, J. J. (2011). Diffusion models of the flanker task: Discrete versus gradual attentional selection. Cognitive Psychology, 63(4), 210-238. https://doi.org/10.1016/j. cogpsych.2011.08.001

Wiegand, K., \& Wascher, E. (2005). Dynamic aspects of stimulusresponse correspondence: Evidence for two mechanisms involved in the Simon effect. Journal of Experimental Psychology:, Human Perception and Performance, 31(3), 453-464. https://doi.org/10. 1037/0096-1523.31.3.453

Wylie, S. A., Ridderinkhof, K. R., Eckerle, M. K., \& Manning, C. A. (2007). Inefficient response inhibition in individuals with mild cognitive impairment. Neuropsychologia, 45(7), 1408-1419. https://doi.org/10.1016/j.neuropsychologia.2006.11.003
Wylie, S. A., van den Wildenberg, W. P., Ridderinkhof, K. R., Bashore, T. R., Powell, V. D., Manning, C. A., \& Wooten, G. F. (2009). The effect of Parkinson's disease on interference control during action selection. Neuropsychologia, 47(1), 145157. https://doi.org/10.1016/j.neuropsychologia.2008.08.001

Zhang, J., \& Kornblum, S. (1997). Distributional analysis and De Jong, Liang, and Lauber's (1994) dual-process model of the Simon effect. Journal of Experimental Psychology: Human Perception and Performance, 23(5), 1543-1551. https://doi.org/10.1037/00 96-1523.23.5.1543

Publisher's note Springer Nature remains neutral with regard to jurisdictional claims in published maps and institutional affiliations. 